\title{
Influencia del procesamiento de materiales cerámicos en su comportamiento como adsorbente de iones en aguas industriales
}

\author{
J. M. VILLORA, P. CALLEJAS, M. F. BARBA \\ Instituto de Cerámica y Vidrio (CSIC). Cantoblanco. Madrid.
}

\begin{abstract}
Las propiedades de un material cerámico están íntimamente relacionadas con el procesamiento seguido para su obtención, dado que éste determina en gran medida su microestructura. En este trabajo se ha diseñado un material cerámico, obtenido a partir de materias primas alternativas (residuos industriales y urbanos), encaminado a retener metales pesados en aguas contaminadas, siguiendo tres procesos distintos: colaje en molde de escayola, prensado uniaxial y extrusión. Para establecer una comparativa entre ellos se han preparado, en los tres casos, en forma de anillos Raschig. Se ha procedido a la caracterización físicoquímica de los materiales así obtenidos, observándose su microestructura mediante microscopía electrónica de barrido, superficie específica (BET), medidas de densidad en mercurio y helio, absorción de agua y porosidad. Asimismo se ha realizado un ensayo dinámico de retención de metales pesados con estos anillos en aguas simuladas, llevando a cabo análisis químicos mediante ICP-OES en las aguas depuradas.
\end{abstract}

Palabras clave: Procesos de conformado, Materiales porosos, Metales pesados, Aguas contaminadas, Anillos Raschig.

\section{Influence of processing on the behaviour of ceramic materials as ion adsorbents in industrial waste waters}

Properties of ceramic materials are directly related with process followed for their production, since the process is determining microstructure. In this work a ceramic material from industrial and urban wastes has been designed and set out for retaining heavy metals from industrial contaminated waters, following three different processes: slip casting, uniaxial pressing and extrusion. These materials have been shaped as Raschig rings to establish a comparison between them, in three cases. A physical-chemical characterisation of the material has been performed. The microstructure was studied by SEM, specific surface area (BET), mercury and helium density measurements, water absorption and porosity. Additionally, a dynamic immobilisation experiment was performed with these rings in simulated waters containing heavy metals. Chemical analyses were carried out by ICP-OES technique in treated waters.

Key words: Forming process, Porous materials, Heavy metals, Waste waters, Raschig Rings.

\section{INTRODUCCIÓN}

La reducción de la contaminación presenta en la actualidad un creciente interés debido a la gran preocupación que existe en todos los niveles de la sociedad por la conservación del medio ambiente. Los procesos de depuración de aguas tratan de convertir los residuos líquidos procedentes del uso de las aguas de abastecimiento en un efluente final aceptable. En los tratamientos terciarios se separan sustancias disueltas que han escapado a tratamientos previos (primarios y secundarios) por precipitación, coagulación, floculación, microfiltración, ultrafiltración, nanofiltración, ósmosis inversa, cambio iónico y adsorción, entre otras operaciones de separación de materias (1).

En la actualidad existen numerosas industrias que generan efluentes que contienen cantidades de metales pesados que no pueden ser directamente vertidos a los cauces de aguas receptoras o reutilizados en diversos usos. El problema que se plantea es conseguir una depuración eficiente mediante un proceso de bajo coste, ya que los tratamientos terciarios suelen presentar costos elevados y dificultades para su puesta en marcha. Por tanto, se está estudiando sobre nuevas alternativas que resulten más económicas (2). En este sentido, la ciencia de materiales cerámicos y vítreos puede aportar soluciones hasta el momento no muy desarrolladas, aunque ya existen trabajos y patentes relacionados con el tema $(3,4)$.

En el presente trabajo se ha preparado un material cerámico que presenta propiedades como adsorbente e intercambiador de iones en aguas contaminadas $(5,6)$. Estos materiales han sido obtenidos por diferentes procesos de conformado con el fin de estudiar cuál de ellos se presenta como el más apropiado económica y funcionalmente, así como plantear la posibilidad de extrapolarlo a escala industrial. Como materias primas de partida se han utilizado tres tipos de residuos procedentes de dos fuentes: urbanas (huesos) e industriales (diatomeas, residuos de la industria papelera). La fase de hidroxiapatito presente en el hueso (7-9) en principio parece ser la responsable de la inmovilización de los metales pesados, aunque pueden actuar otros mecanismos, aún no esclarecidos en su totalidad (10-16).

\section{MATERIALES Y MÉTODOS}

\subsection{Materias primas}

Las materias primas empleadas en la preparación del material son residuos urbanos e industriales: harina de huesos de animales procedentes del consumo de carnes, residuos de la industria del papel (denominados lodos), y filtros de diatomeas utilizados y desechados en la industria cervecera. La harina de huesos (suministrada por ICN Biomedicals, USA) fue calcinada a $1000^{\circ} \mathrm{C} / 24 \mathrm{~h}$ para eliminar la materia orgánica. El resto de las materias primas sufrieron otro proceso de acondicionamiento previo a su utilización. Los lodos de papelera (proporcionados por Lemona Industrial S.A, España) se pasaron por un tamiz de luz de malla de $500 \mu \mathrm{m}$ y se secaron a $110^{\circ} \mathrm{C}$ durante $24 \mathrm{~h}$. Las diatomeas (facilitadas por Mahou, España) se secaron igualmente a $110^{\circ} \mathrm{C} / 24 \mathrm{~h}$ y posteriormente se calcinaron a $1000^{\circ} \mathrm{C} / 24 \mathrm{~h}$ con el objeto de eliminar los restos de materia orgánica. 


\subsection{Aditivos}

En cada proceso seguido se han utilizado aditivos para lograr una mayor reproducibilidad de los productos obtenidos, además de conseguir una adecuada resistencia mecánica en verde. Dado que todos los aditivos empleados fueron orgánicos y por tanto eliminados por combustión durante el tratamiento térmico, no alteraron la composición química del material.

En el proceso de colaje en molde de escayola se ha utilizado como dispersante un poliacrilato sódico (Dispex-N 40, Allied Colloids, USA) en una proporción del $0,25 \%$ en peso y un ligante, carboximetil celulosa (Optapix C 1000 G, Zschimmer \& Schwarz, Alemania) en un 2\%.

Las pastas para extrusión se prepararon mediante la adición de un plastificante polisacárido (Zusoplast PS-1, Zschimmer \& Schwarz, Alemania) en un 2,5\% en peso relativo a la cantidad de sólidos. No se utilizaron lubricantes ya que no se observaron dificultades ni irregularidades en la salida de la pasta por la boquilla.

El polvo de prensado se preparó añadiendo un 10\% de humedad y un alcohol polivinílico como ligante, (Optapix PAF 35, Zschimmer \& Schwarz, Alemania) al 1\%. Fue necesario el empleo de un aceite lubricante con el fin de facilitar el desmoldeo de los anillos prensados.

\subsection{Formulación del material}

Se pretendió obtener un material con alta porosidad dentro del sistema de óxidos mayoritarios $\mathrm{P}_{2} \mathrm{O}_{5}-\mathrm{CaO}-\mathrm{SiO}_{2}$, con un contenido en peso de $\mathrm{SiO}_{2}>50 \%$ y una relación de óxidos $\mathrm{CaO} / \mathrm{P}_{2} \mathrm{O}_{5} \geq 2$. Para ello se han empleado las materias primas en las siguientes proporciones (\% en peso): 49,3 de diatomeas (como fuente de $\mathrm{SiO}_{2}$ ), 33,4 de hueso calcinado (que contiene la fase hidroxiapatito $\mathrm{Ca}_{5}\left(\mathrm{PO}_{4}\right)_{3} \mathrm{OH}$ ) y 17,3 de lodo (que aporta $\mathrm{CaCO}_{3}$ ). El uso de diatomeas aporta porosidad intragranular además de un componente (la sílice) que mejora las propiedades mecánicas del material. El uso de carbonato cálcico está justificado debido a que a) con este exceso de Ca, se evita la descomposición térmica del hidroxiapatito presente en el hueso $(13,17)$ y b) los carbonatos producen porosidad en el material al descomponerse por acción de la temperatura y liberar $\mathrm{CO}_{2}$; este gas crea canales en el material, produciendo así una porosidad interconectada.

Las materias primas se molieron y homogeneizaron en medio acuoso, en un molino de atrición con bolas de alúmina durante $30 \mathrm{~min}$.

\subsection{Fabricación de los anillos Raschig}

Se han obtenido anillos Raschig (cilindros huecos de igual diámetro exterior que altura) por los diferentes procesos de conformado anteriormente mencionados: colaje en molde de escayola, prensado uniaxial y extrusión. Para que las dimensiones de estos anillos no variaran significativamente entre procesos, fue preciso adecuar todas las medidas de los moldes y boquillas, obteniendo piezas comprendidas entre los siguientes intervalos: diámetro exterior y altura: $8,0 \pm 0,2 \mathrm{~mm}$ y espesor de pared del anillo: 2,0 $\pm 0,3 \mathrm{~mm}$.

Los moldes utilizados para el colaje se fabricaron con escayola Alamo 70, con una proporción de agua/escayola de 55/100 en peso. Como contramolde se utilizó una varilla de vidrio de $10 \mathrm{~mm}$ de diámetro y $20 \mathrm{~cm}$ de longitud. El tiempo de formación de pared fue de $15 \mathrm{~min}$. La barbotina con las adecuadas propiedades reológicas, que contenía un $50 \%$ en peso de sólidos, se coló sobre el molde y transcurrido ese tiempo se eliminó por vertido el exceso de la misma. Se dejó endurecer hasta que la contracción por secado permitió su desmoldeo.
Estos tubos así obtenidos fueron sometidos al tratamiento térmico.

Para la extrusión de la pasta se utilizó una extrusora vertical de pistón, accionada manualmente, con una boquilla de diámetro exterior 9,5 mm e interior 5,0 mm. La pasta, con un $40 \%$ en peso de agua, fue introducida por su parte superior y por la inferior, donde está situada la boquilla, se obtuvieron tubos de $20 \mathrm{~cm}$ de longitud aproximada. Estos tubos se secaron en una estufa a $60^{\circ} \mathrm{C}$ con el fin de producir una eliminación homogénea del agua y evitar la aparición de grietas o deformaciones al contraer. Los tubos una vez secos se sometieron al ciclo de cocción.

En el caso del prensado uniaxial se utilizó un molde y una máquina de prensado industriales. Los anillos que se prensaron poseían las siguientes dimensiones en verde: diámetro exterior $9,3 \mathrm{~mm}$, igual que su altura y diámetro interior: 5,4 $\mathrm{mm}$. Se consiguieron 26 anillos en cada prensada. La máquina se ajustó para que la longitud del anillo igualara a su altura, pero no se pudo llevar a acabo ningún control de la presión aplicada.

\subsection{Sinterización}

El tratamiento térmico aplicado al material una vez conformado fue diseñado a partir de ensayos de dilatometría y microscopía de calefacción. Se realizó un ciclo de cocción que alcanzó como temperatura máxima $1150^{\circ} \mathrm{C}$ durante $1 \mathrm{~h}$, con una velocidad de calentamiento y enfriamiento de $6^{\circ} \mathrm{C} / \mathrm{min}$, en un horno eléctrico (SATER, España) con atmósfera de aire. Siguiendo este tratamiento térmico se logran buenas propiedades mecánicas en el material una vez cocido (18) y se mantiene la fase de hidroxiapatito al final del mismo (19).

\subsection{Métodos de caracterización}

\subsubsection{PROPIEDADES FÍSICAS DE PASTAS Y BARBOTINAS}

Las cantidades de aditivos utilizados en el proceso de colaje fueron determinadas realizando medidas de viscosidad con un viscosímetro rotacional de velocidad controlada (modelo Brookfield LVDV-I+, USA) en las suspensiones preparadas, fijando el contenido de sólidos y variando las cantidades de aditivos. También se estudió el comportamiento del fluido midiendo viscosidades en función de la velocidad de cizalla

En las pastas de extrusión, la proporción de aditivo óptima se determinó mediante los procedimientos de caracterización de arcillas basados en la determinación del índice de plasticidad, límite líquido (Norma UNE 103-103-94) y límite plástico (Norma UNE 103-104-93). Para el primero de ellos fue preciso el empleo del aparato de Casagrande: un utensilio mecánico que consiste en una cuchara de aleación de cobre, suspendida de un dispositivo diseñado para controlar su caída sobre una base dura. (20)

\subsubsection{MEDIDA DE DENSIDADES, POROSIDADES Y TAMAÑO DE PARTÍCULA}

La densidad aparente (D Hg) del material tanto en verde como sinterizado, se determinó por inmersión en mercurio de probetas obtenidas por los tres procesos basándose en el principio de Arquímedes, sumergiendo la probeta en un recipiente con mercurio y midiendo el peso del mismo que desaloja.

Las medidas de densidad real (D He) del material sinterizado se realizaron en un picnómetro de helio, modelo Multipycnometer 
(QuantaChrome, USA).

La porosidad abierta del material sinterizado $\left(\mathrm{P}_{1}\right)$ se calculó mediante la fórmula:

$$
\mathrm{P}_{1}=[(\mathrm{D} \mathrm{He}-\mathrm{D} \mathrm{Hg}) / \mathrm{D} \mathrm{He}] \times 100
$$

Como método comparativo, se calculó también la porosidad abierta en las probetas sinterizadas $\left(\mathrm{P}_{2}\right)$ a través de las medidas de capacidad de absorción de agua (Caa) y densidad aparente (Dap) del material en agua. En este caso fue necesario el empleo de una balanza hidrostática. Todos estos ensayos se llevaron a cabo según la norma UNE-EN ISO 10545-3:1997.

Las fórmulas empleadas en estos cálculos fueron:

Caa $=($ Peso probeta húmeda - Peso probeta seca $) /$ Peso probeta seca

Dap $=$ Peso probeta seca $/($ Peso probeta húmeda - Peso probeta sumergida $)$ [3]

$\mathrm{P}_{2}=$ Caa $\times$ Dap

La superficie específica (BET) de los materiales sinterizados y del polvo de partida se determinó en un equipo Monosorb (QuantaChrome, USA) que emplea $\mathrm{N}_{2}$ líquido como adsorbato y un portamuestras especial que permite introducir cuerpos del tamaño de los anillos Raschig fabricados.

La distribución granulométrica de la mezcla de partida se determinó con un analizador LASER de partículas (Mastersizer, Malvern, UK)

\subsubsection{MICROSCOPÍA ELECTRÓNICA DE BARRIDO}

La microestructura de los materiales fue observada utilizando un microscopio electrónico de barrido modelo C. Zeiss DSM-950 equipado con un equipo Tracor Nortthem para microanálisis por dispersión de energías. Las muestras fueron preparadas en fractura reciente.

\subsubsection{ENSAYOS DE RETENCIÓN}

El ensayo llevado a cabo consistió en depurar 5 litros de una disolución de $10 \mathrm{mg} / \mathrm{l}$ de $\mathrm{Cd}$ y Pb, haciéndolos recircular mediante una bomba en cuya cámara de $120 \mathrm{~cm}^{3}$ de capacidad se encontraban los anillos de los materiales obtenidos por distintos procesos. Se tomaron muestras de $100 \mathrm{ml}$ cada media hora hasta 5 horas y otra muestra a las 10 horas. El ensayo se repitió para cada clase de anillos. Dado que éstos presentan diferentes densidades, según el proceso de obtención seguido, el peso de los mismos fue diferente: $54,66 \mathrm{~g}$ para colaje, 67,17 $\mathrm{g}$ para extrusión y $72,86 \mathrm{~g}$ para el prensado.

El análisis de las disoluciones se realizó mediante ICP-OES en un espectrómetro para análisis simultáneo con detector de estado sólido Thermo Jarrel Ash modelo IRIS ADVANTAGE (USA).

\section{RESULTADOS Y DISCUSIÓN}

\subsection{Medidas de porosidad}

Los resultados obtenidos acompañados de su desviación estándar (calculada con un mínimo de 7 repeticiones) en las determinaciones de a) densidad real en las probetas sinterizadas (D He) y densidad aparente $(\mathrm{D} \mathrm{Hg})$ tanto en verde como sinterizadas, b) porosidad abierta $\left(\mathrm{P}_{1}\right.$, fórmula [1]), c) capacidad de absorción de agua (fórmula [2]), d) densidad aparente (fórmula [3]) y e) porosidad abierta $\left(\mathrm{P}_{2}\right.$, fórmula [4]) se presentan en la Tabla 1.
TABLA 1. - RESUlTADOS DE LAS MEDIDAS DE DENSIDAD, CAPACIDAD DE ABSORCIÓN DE AGUA Y POROSIDAD

\begin{tabular}{|l|c|c|c|}
\hline & COLAJE & EXTRUSIÓN & PRENSADO \\
\hline Densidad aparente en verde $\mathbf{( H g )}$ & $0,84 \pm 0,04$ & $1,03 \pm 0,03$ & $1,4 \pm 0,2$ \\
\hline Densidad aparente sinterizado (Hg) & $1,1 \pm 0,1$ & $1,4 \pm 0,1$ & $1,84 \pm 0,04$ \\
\hline Densidad real sinterizado (He) & $2,73 \pm 0,01$ & $2,68 \pm 0,01$ & $2,82 \pm 0,01$ \\
\hline$\%$ Porosidad & $\mathbf{5 9} \pm 4$ & $\mathbf{4 8} \pm 4$ & $\mathbf{3 5} \pm 1$ \\
\hline Capac. Absorc. Agua & $41 \pm 7$ & $30 \pm 5$ & $18 \pm 2$ \\
\hline Densidad aparente en agua & $1,4 \pm 0,2$ & $1,57 \pm 0,08$ & $1,84 \pm 0,07$ \\
\hline$\%$ Porosidad & $\mathbf{5 7} \pm \mathbf{3}$ & $\mathbf{4 6} \pm \mathbf{5}$ & $\mathbf{3 2 \pm 2}$ \\
\hline
\end{tabular}

Se observa que los anillos obtenidos por el proceso de colaje tienen menor densidad en verde que los extrusionados, seguidos de los obtenidos por prensado. Estas diferencias son debidas al mayor o menor grado de empaquetamiento que se consiguen con cada proceso. La presión que se aplica en el prensado ha sido mayor que en la extrusión, y éstas a su vez mayores que la presión de succión que ejercen los moldes de escayola en el colaje. Esta misma tendencia se mantiene para las piezas una vez sinterizadas. Los métodos de medida de las densidades en sinterizado están basados en el mismo fundamento (principio de Arquímedes) pero por inmersión en dos líquidos distintos: agua y mercurio. El método que usa mercurio ofrece resultados ligeramente inferiores. Las diferencias entre los dos métodos se hacen menores a medida que disminuye la porosidad del material, hasta el punto que en el caso del prensado (el menos poroso) coinciden. Los datos resultantes de porosidad varían en sentido inverso al de las densidades (colaje $>$ extrusión $>$ prensado). Como cabría esperar al tratarse de materiales con la misma composición y tratamiento térmico, las densidades reales son muy semejantes.

Las diferencias entre estos dos métodos (que quedan minimizadas en los errores aleatorios) tienen su explicación debido a que el mercurio tiene mayor tensión superficial y menor ángulo de mojado con el material cerámico que el agua, por lo que cuantifica mayor volumen y por tanto, menor densidad y mayor porosidad; existen poros a los cuales el mercurio le es imposible acceder a la presión que se ejerce por la inmersión.

El empleo de estos dos métodos comparativos queda justificado si se tiene en cuenta que el material va a estar en contacto con agua, de manera que la porosidad medida en este medio dará una idea más cercana a la accesibilidad de la misma al material.

\subsection{Medidas de superficie específica y tamaño de partícula}

El tamaño medio de partícula determinado para la mezcla de las materias primas posterior a su molienda y homogeneizado en molino de atrición fue de $6,6 \mu \mathrm{m}$, estando el $90 \%$ de la muestra por debajo de $22 \mu \mathrm{m}$. La superficie específica encontrada para este polvo fue de 18,5 $\mathrm{m}^{2} / \mathrm{g}$.

Se realizaron determinaciones de superficie específica en el material sinterizado, obtenido por los distintos métodos de conformado, encontrándose que para el proceso de colaje fue de $0,7 \mathrm{~m}^{2} / \mathrm{g}$, para el de extrusión $0,6 \mathrm{~m}^{2} / \mathrm{g}$ y por último, para el prensado, de $0,2 \mathrm{~m}^{2} / \mathrm{g}$, afectados por un error de $\pm 0,1$. Los datos de colaje y extrusión no difieren significativamente, no así el prensado, que muestra un área superficial notablemente menor. Estos resultados varían en el mismo sentido que las porosidades mencionadas en el apartado anterior. En este material, un aumento de la porosidad abierta se refleja en un incremento de la superficie específica. 


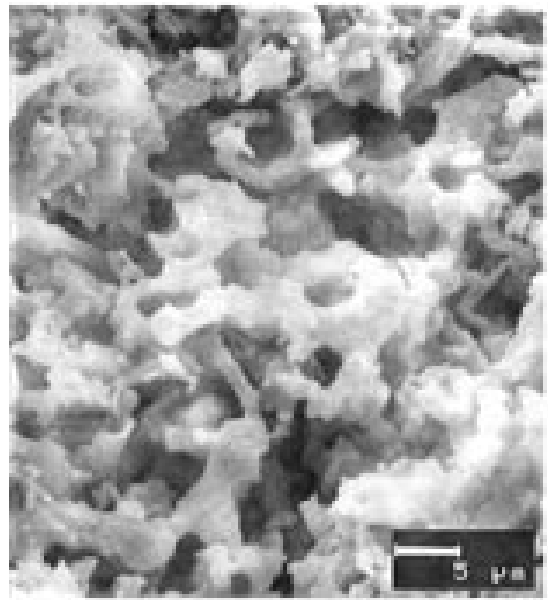

Colaje $\times 2.000$

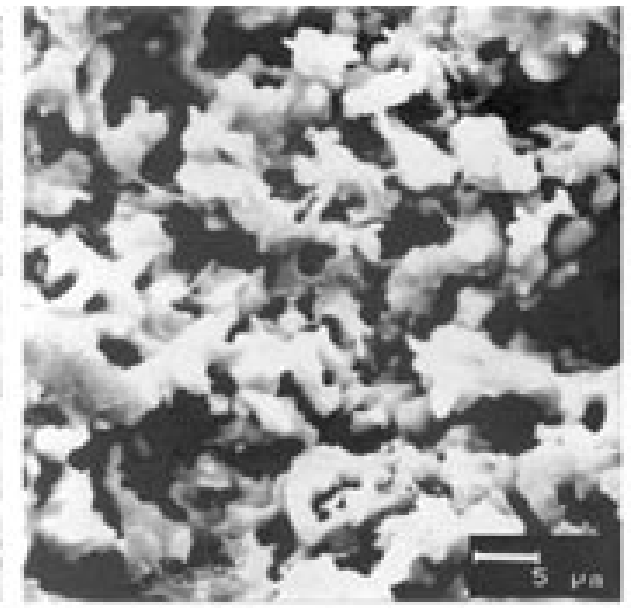

Extrusión x 2.000

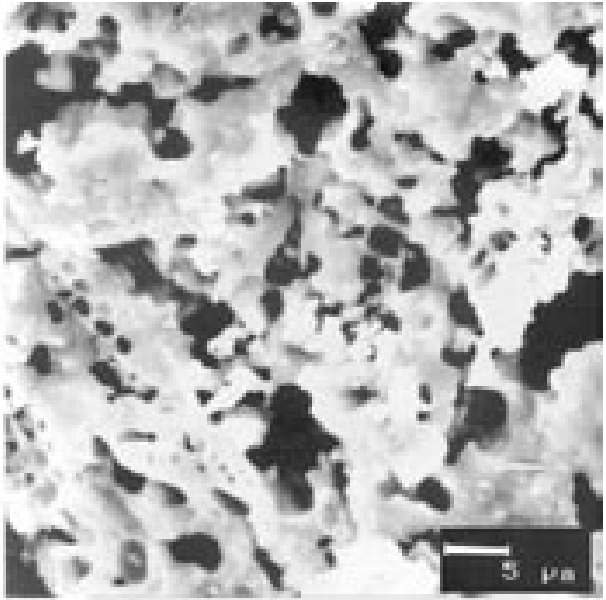

Prensado x 2.000

Fig. 1. - Microestructura observada por MEB del material conformado por los tres procesos

\subsection{Microscopía Electrónica de Barrido (MEB)}

La microestructura de los materiales obtenidos fue estudiada por MEB sobre fractura reciente (Fig. 1). Es muy homogénea, con abundantes poros pequeños interconectados por canales, que pueden apreciarse junto a otros de mayor tamaño, redondeados y aislados que aparecen esporádicamente. También se observa un menor grado de sinterización en el caso del colaje.

\subsection{Ensayos de retención}

En las gráficas de la Fig. 2 se representan los porcentajes de retención frente al tiempo de $\mathrm{Cd}$ y $\mathrm{Pb}$. Puede observarse que la tendencia de las curvas es similar en el caso del cadmio, alcanzándose prácticamente los mismos niveles de retención para los materiales obtenidos por los tres procesos. Se consiguen mayores porcentajes de retención en el caso del plomo aún en el proceso de prensado que es el que a cualquier tiempo ofrece menores valores. Se observa que los materiales, a las $10 \mathrm{~h}$, continúan reteniendo metales pesados, por lo tanto, no se llegaron a saturar completamente.

Teniendo en cuenta los resultados obtenidos, podría discutirse si el material presenta más afinidad por un elemento que por otro. Para poder hacer una comparación, es necesario transformar la masa retenida en moles (que da una idea más clara de la cantidad de iones metálicos retenidos en el material) y referirlos al peso del material que se utiliza en cada experimento, ya que este factor variaba entre los procesos, al tener los anillos diferentes densidades. En la Tabla 2 se muestran los miligramos y moles retenidos para cada elemento en cada proceso referidos a gramo de material así como la suma de ambos a las $10 \mathrm{~h}$ de depuración. Con el proceso de colaje se retienen más moles de $\mathrm{Pb}$ que de $\mathrm{Cd}$; esta tendencia se mantiene en menor proporción para el proceso de extrusión y se invierte en el caso del prensado. Considerando que el material tiene la misma composición en los tres casos y la afirmación anterior, no parece que exista una clara preferencia del material por ninguno de los dos elementos. Sin embargo, sí se aprecian diferencias significativas en cuanto a las cantidades retenidas en cada material obtenido por los distintos métodos. Concretamente, en el caso de colaje es donde se consigue una mayor retención, seguido de la extrusión y por último, el prensado. Estos resultados coinciden con la tendencia observada en las determinaciones de porosidad y superficie específica, es decir, los materiales más porosos y de mayor superficie específica son los que más metales pesados retienen. Por tanto, estos
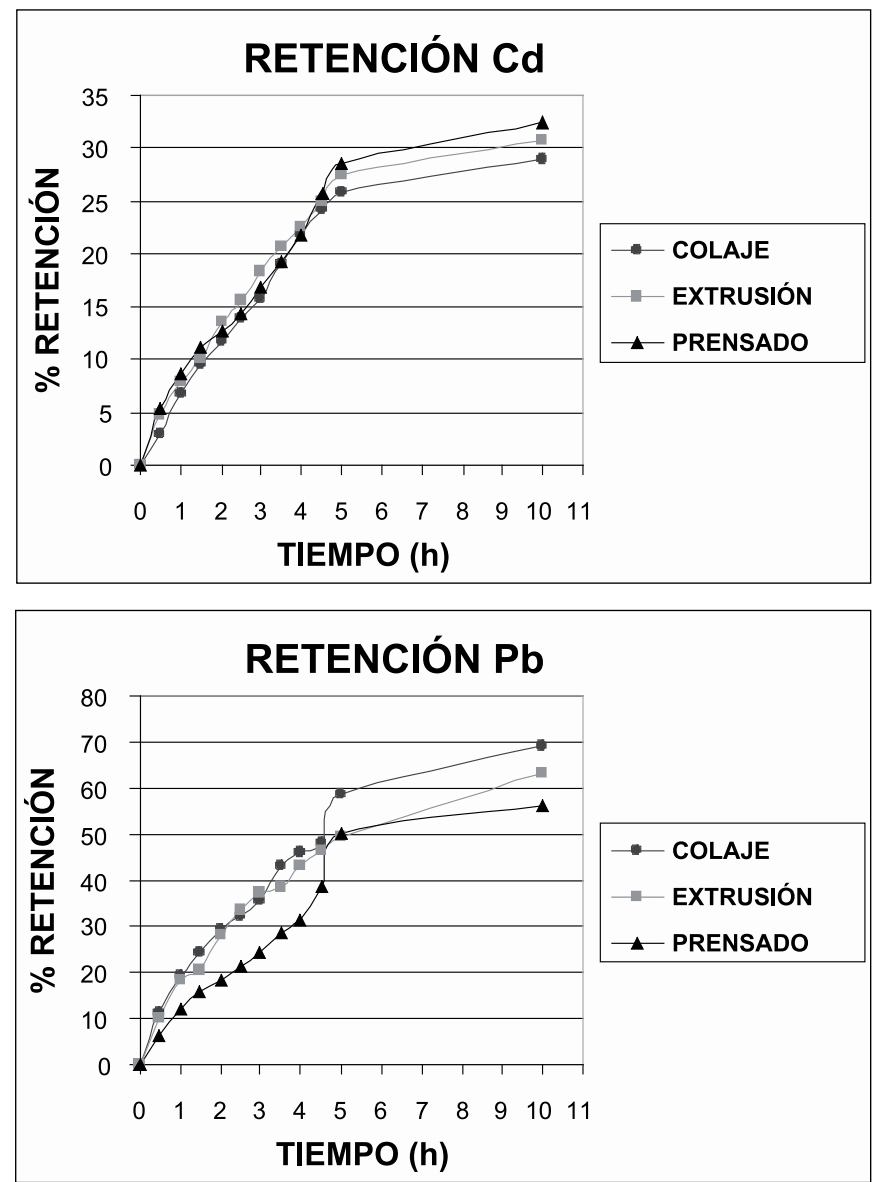

Fig. 2. - Porcentajes de retención de $\mathrm{Cd}$ y $\mathrm{Pb}$ en los materiales frente al tiempo.

TABLA 2. - CANTIDADES DE METALES PESADOS RETENIDOS POR GRAMO DE MATERIAL EN CADA PROCESO A LAS $10 \mathrm{H}$

\begin{tabular}{|l|c|c|c|}
\hline & COLAJE & EXTRUSIÓN & PRENSADO \\
\hline mg Cd retenidos/g material & 0,260 & 0,228 & 0,225 \\
\hline $\mathrm{mg} \mathrm{Pb}$ retenidos/g material & 0,629 & 0,463 & 0,379 \\
\hline $\mathrm{mg} \mathrm{Cd}+\mathrm{Pb}$ retenidos/g material & $\mathbf{0 , 8 8 9}$ & $\mathbf{0 , 6 9 1}$ & $\mathbf{0 , 6 0 4}$ \\
\hline moles Cd retenidos/g material & $2,31 \cdot 10^{-3}$ & $2,02 \cdot 10^{-3}$ & $2,00 \cdot 10^{-3}$ \\
\hline moles Pb retenidos/g material & $3,04 \cdot 10^{-3}$ & $2,23 \cdot 10^{-3}$ & $1,83 \cdot 10^{-3}$ \\
\hline moles Cd + Pb retenidos/g material & $\mathbf{5 , 3 5 \cdot 1 0 ^ { - 3 }}$ & $\mathbf{4 , 2 5 \cdot 1 0 ^ { - 3 }}$ & $\mathbf{3 , 8 3 \cdot 1 0 ^ { - 3 }}$ \\
\hline
\end{tabular}


hechos confirman que las propiedades del material como inmovilizador de estos elementos se muestran en su superficie activa, o área del material que se encuentra en contacto con el agua.

\section{CONCLUSIONES}

El estudio de los procesos de fabricación y la caracterización de los anillos Raschig del material formulado en este trabajo, así como los ensayos realizados con ellos en aguas simuladas, han dado lugar a las siguientes conclusiones:

- Los tres métodos de conformado seguidos han dado lugar a materiales en forma de anillos Raschig con adecuada porosidad y superficie específica para ser aplicados como inmovilizadores de metales pesados en aguas contaminadas.

- Los parámetros más importantes a controlar para maximizar la retención de los elementos ensayados son la porosidad y la superficie específica, ya que al aumentar éstos se eleva el grado de retención en el material.

- El método de colaje se presenta como el más adecuado en términos de capacidad de retención, pero económicamente es el menos favorable debido a que requiere el uso de más aditivos y genera residuos de los moldes utilizados.

- Los métodos de extrusión y prensado son más favorables para su fabricación a nivel industrial. En el caso del prensado será necesario un acondicionamiento previo del polvo de prensado para un correcto llenado de los moldes.

\section{AGRADECIMIENTOS}

A la CICYT por la financiación (proyecto AMB99-1187 y DP120000153-P4-0) y a las empresas Mahou S.A. y ESMALTES S.A. por el suministro de parte de las diatomeas y seguimiento activo del proyecto.

\section{BIBLIOGRAFÍA}

1. American Water Works Association Research Foundation, Research Fundation Lyonnaise des Eaux, Water Research Commission of South Africa: «Tratamiento del agua por procesos de membrana. Principios, procesos y aplicaciones». Edit. McGraw Hill. Aravaca, Madrid (2002).

2. J. A. Pérez y M. Espigares, «Estudio sanitario del agua», Edit. Universidad de Granada. Granada (1995)
3. Sandu, Ion; Nitescu, Eftimie; Calu, Nicolae; Berdan, Ioan; Smcot, Rodica; Bialus, Amediu; Popa, Grabiel; Viscu, Vasile; Stanila, Alexandru: Method and apparatus for producing drinking water. Patente No. Ro 77382.

4. Schott information, $n^{\circ} 75$, Septiembre 1995: Vidrio de poros abiertos Siporax para el tratamiento terciario de aguas. Pag. 13-15.

5. A.M. García, J.M. Villora, D.A. Moreno, C. Ranninger, P.Callejas, M.F. Barba. "Heavy Metals Bioorremediation from Polluted Water by Glass-Ceramic Materials" J.Am.Ceraam.Soc., 86 [12]2.200-202 (2003)

6. A.M. García, J.M. Villora, D.A. Moreno, C. Ranninger, P. Callejas, M.F. Barba. "Aplicación de un material vitrocerámico a la biorremediación de metales pesados". Bol.Soc.Esp.Ceram.V., 43 [1] 67-70 (2004)

7. N. Ö. Engin and A. C. Tas. «Preparation of Porous $\mathrm{Ca}_{10}\left(\mathrm{PO}_{4}\right)_{6}(\mathrm{OH})_{2}$ and $\beta$ $\mathrm{Ca}_{3}\left(\mathrm{PO}_{4}\right)_{2}$ Bioceramics». J. Am. Ceram. Soc. 83 [7] 1581-84 (2000)

8. T. M. G. Chu, J. W. Halloran, S. J. Hollister, S. E. Feinberg. «Hydroxyapatite implants with designed internal architecture». J. Mater. Sci.: Mater. Med. 12 471-478 (2001).

9. C. G. Portillo. «Composición, propiedades y aplicaciones de la porcelana de huesos. Silicatos 9 17-22 (1998).

10. Q. Y. Ma, T. J. Logan, and S. J. Traina. «Effects of $\mathrm{NO}^{3-}, \mathrm{Cl}^{-}, \mathrm{F}^{-}, \mathrm{SO}_{4}{ }^{2-}$, and $\mathrm{CO}_{3}{ }^{2-}$ on $\mathrm{Pb}^{2+}$ Immovilization by Hydroxyapatite». Environ. Sci. Technol. 28 [3] 408-418 (1994).

11. Q. Y. Ma, T. J. Logan, S. J. Traina and J. A. Ryan «Effects of Aqueous Al, Cd, $\mathrm{Cu}, \mathrm{Fe}(\mathrm{II}), \mathrm{Ni}$, and $\mathrm{Zn}$ on $\mathrm{Pb}$ Immovilization by Hydroxyapatite». Environ. Sci. Technol. 28 [7] 1219-1228 (1994).

12. S. Suzuki, T. Fuzita, T. Maruyama, M. Takahashi, Y. Hikichi. «CationExchange Characterics of Sintered Hydroxyapatite in the Strongly Acidic Region». J. Amer. Ceram. Soc. 76 [6] 1638-40 (1993)

13. S. Suzuki, K. Itoh, M. Ohgaki, M. Ohtani, M. Ozawa. «Preparation of sintered filter for ion exchange by a doctor blade method with aqueous slurries of needlelike hydroxyapatite». Ceram. Int. 25 287-291 (1999).

14. J. G. P. Binner and J. Reichert. « Hydroxyapatite filters for the removal of heavy metals ions from aqueous solutions». Br. Ceram. Proc. 55 (21st Century Ceramics) 63-78 (1996).

15. J. Reichert, J. G. P. Binner. «An evaluation of hydroxyapatite-based filters for removal of heavy metal ions from aqueous solutions». J. Mater. Sci. 31 12311241 (1996).

16. S. Furuta, H. Katsuki and S. Komarneni. «Removal of Lead Ions Using Porous Hydroxyapatite Monoliths Synthesized from Gypsum Waste». J. Ceram. Soc. Japan 108 [3] 315-317 (2000)

17. J. M. Villora, P. Callejas, M. F. Barba «Métodos de síntesis y comportamiento térmico del Hidroxiapatito» Bol. Soc. Esp. Ceram. V., 41 [5] 443-450 (2002)

18. P. Callejas, J. M. Villora, F. Barba: «Procesos de obtención de anillos cerámicos Raschig aplicados al tratamiento de aguas residuales». XI Congreso Internacional de Industria, Minería y Metalurgia. Zaragoza, Junio 2002.

19. J. M. Villora, P. Callejas M. F. Barba. "Processing of highly porous hydroxyapatite from waste raw materials» Key Engineering Materials, Vols 206-213, pp. 895-898 (2002).

20. E. Gippini «Pastas Cerámicas» Libro publicado bajo los auspicios de la Sociedad Española de Cerámica y Vidrio, Madrid, 1979. pp. 51-72.

Recibido: 01.02 .03

Aceptado: 30.11 .03 